\title{
Clinical and genetic differences between bipolar disorder type 1 and 2 in multiplex families
}

\author{
Jose Guzman-Parra', Fabian Streit $\mathbb{1}^{2}$, Andreas J. Forstner $\mathbb{B}^{3,4}$, Jana Strohmaier $\mathbb{D}^{2}$, Maria José González \\ Susana Gil Flores ${ }^{6}$, Francisco J. Cabaleiro Fabeiro ${ }^{7}$, Francisco del Río Noriega ${ }^{8}$, Fermin Perez Perez ${ }^{5}$, \\ Jesus Haro González ${ }^{9}$, Guillermo Orozco Diaz ${ }^{10}$, Yolanda de Diego-Otero (10 ${ }^{1}$, Berta Moreno-Kustner ${ }^{11}$, \\ Georg Auburger ${ }^{12}$, Franziska Degenhardt ${ }^{4}$, Stefanie Heilmann-Heimbach ${ }^{4}$, Stefan Herms (1), ${ }^{4,}$, Per Hoffmann 4,13,14, \\ Josef Frank $\mathbb{1}^{2}$, Jerome C. Foo $\mathbb{1}^{2}$, Lea Sirignano $\mathbb{B}^{2}$, Stephanie H. Witt $\mathbb{1}^{2}$, Sven Cichon ${ }^{13,14}$, Fabio Rivas ${ }^{1}$, \\ Fermín Mayoral', Markus M. Nöthen ${ }^{4}$, Till F. M. Andlauer $\mathbb{1}^{15}$ and Marcella Rietschel $\mathbb{1}^{2}$
}

\begin{abstract}
The two major subtypes of bipolar disorder (BD), BD-I and BD-II, are distinguished based on the presence of manic or hypomanic episodes. Historically, BD-II was perceived as a less severe form of BD-I. Recent research has challenged this concept of a severity continuum. Studies in large samples of unrelated patients have described clinical and genetic differences between the subtypes. Besides an increased schizophrenia polygenic risk load in BD-l, these studies also observed an increased depression risk load in BD-II patients. The present study assessed whether such clinical and genetic differences are also found in BD patients from multiplex families, which exhibit reduced genetic and environmental heterogeneity. Comparing 252 BD-I and 75 BD-II patients from the Andalusian Bipolar Family (ABiF) study, the clinical course, symptoms during depressive and manic episodes, and psychiatric comorbidities were analyzed. Furthermore, polygenic risk scores (PRS) for BD, schizophrenia, and depression were assessed. BD-I patients not only suffered from more severe symptoms during manic episodes but also more frequently showed incapacity during depressive episodes. A higher BD PRS was significantly associated with suicidal ideation. Moreover, BD-I cases exhibited lower depression PRS. In line with a severity continuum from BD-II to BD-I, our results link BD-I to a more pronounced clinical presentation in both mania and depression and indicate that the polygenic risk load of BD predisposes to more severe disorder characteristics. Nevertheless, our results suggest that the genetic risk burden for depression also shapes disorder presentation and increases the likelihood of BD-II subtype development.
\end{abstract}

\section{Introduction}

Bipolar disorder (BD) is a severe, highly heritable mental disorder characterized by fluctuations in mood state and energy with recurring episodes of depression altering with

\footnotetext{
Correspondence: Till F. M. Andlauer (till.andlauer@tum.de)

'Department of Mental Health, University Regional Hospital of Málaga, Institute of Biomedicine of Málaga (IBIMA), Málaga, Spain

${ }^{2}$ Department of Genetic Epidemiology in Psychiatry, Central Institute of Mental Health, Medical Faculty Mannheim, Heidelberg University, Mannheim, Germany

Full list of author information is available at the end of the article

These authors contributed equally: Jose Guzman-Parra, Fabian Streit, Till F. M. Andlauer, Marcella Rietschel
}

episodes of mania or hypomania. Its early onset, chronicity, high prevalence (of approximately $1 \%$ ), and lack of optimal treatment renders it one of the world's most disabling conditions ${ }^{1}$. BD is etiologically heterogeneous, and the current diagnostic classification describes two major subtypes, BD type I (BD-I) and II (BD-II), which differ by the absence of full-blown manic episodes in BDII. BD-I can be diagnosed based on a single manic episode, yet, in most cases, depressive episodes also occur. BD-II is diagnosed if at least one hypomanic and one depressive episode have occurred. Hypomanic episodes are, by definition, clinically less severe than manic ones, potentially

\section{(c) The Author(s) 2021}

\footnotetext{
(c) Open Access This article is licensed under a Creative Commons Attribution 4.0 International License, which permits use, sharing, adaptation, distribution and reproduction cc) in any medium or format, as long as you give appropriate credit to the original author(s) and the source, provide a link to the Creative Commons license, and indicate if changes were made. The images or other third party material in this article are included in the article's Creative Commons license, unless indicated otherwise in a credit line to the material. If material is not included in the article's Creative Commons license and your intended use is not permitted by statutory regulation or exceeds the permitted use, you will need to obtain permission directly from the copyright holder. To view a copy of this license, visit http://creativecommons.org/licenses/by/4.0/.
} 
have a shorter duration, are not characterized by a marked impairment in social or occupational functioning, and do not require hospitalization; the occurrence of any psychotic symptoms qualifies an episode as manic ${ }^{2}$.

Until recently, it was thought that both $\mathrm{BD}$ subtypes could be classified along a spectrum of the affective disorders defined by the extent and severity of mood elevation (i.e., major depressive disorder (MDD) $<\mathrm{BD}-\mathrm{II}<\mathrm{BD}$ I). This concept is in line with findings of BD-I patients suffering from more psychotic ${ }^{3-6}$ and melancholic symptoms $^{3}$, more hospitalizations ${ }^{6-8}$, and more severe and widespread impairment of cognitive functions ${ }^{9}$. It is also in line with the observation in several previous studies, that risk for either subtype of $\mathrm{BD}$ is higher for those with relatives affected by BD-I (compared to MDD and $\mathrm{BD}-\mathrm{II})^{2,10,11}$. However, this concept has been challenged by a series of studies which report a higher total number of episodes ${ }^{12-14}$, increased comorbidity with anxiety disorders $^{8,13,14}$ and personality disorders ${ }^{8,15}$, as well as lower functioning ${ }^{15}$ and quality of life ${ }^{16}$ in BD-II compared to BD-I patients, although these findings were not consistent across studies ${ }^{7,8,17,18}$.

Recent large-scale formal and molecular genetic studies reported a higher heritability of BD-I than of BD-II ${ }^{11,19-21}$ and a high genetic correlation between BD-I and BD-II ${ }^{19-21}$. While the difference in heritability and the high genetic correlation between the subtypes may argue in favor of a severity continuum ${ }^{21}$, other findings instead point to partially distinct genetic etiologies of BD-I and BD-II. In contrast to other previous studies ${ }^{2,10,11}$, a large-scale Swedish registry-based family study found that relatives of $\mathrm{BD}$ patients showed the highest risk for the respective $\mathrm{BD}$ subtype: relatives of patients with BD-I had an increased risk for BD-I compared to BD-II and relatives of patients with BD-II had a trend for a higher risk for BD-II ${ }^{20}$. Different patterns of familial co-aggregation, genetic correlation, and polygenic risk score (PRS) analyses of both subtypes with other psychiatric disorders, in particular of BD-I with schizophrenia (SCZ) and of BD-II with MDD, underline potential molecular differences between the two subtypes $^{11,19-24}$.

Clinical differences between BD-I and BD-II cases have rarely been studied in multiplex families with a high density of BD cases. An advantage of such multiplex families is the reduced genetic and environmental heterogeneity compared to unrelated case/control cohorts ${ }^{25}$. In a previous study on BD multiplex families, Frankland et al. ${ }^{26}$ described more mixed states in BD-II and more psychomotor retardation as well as more psychotic features of depressive episodes in BD-I cases. We have previously shown that, compared to unaffected family members and unrelated controls, BD patients from multiplex families have a high genetic risk load specifically for $\mathrm{BD}^{27,28}$. Furthermore, family members also had a higher genetic risk load for SCZ and, to a lesser degree, for MDD than unrelated controls ${ }^{28}$. Therefore, we hypothesize that a correlation between the genetic risk burden for psychiatric disorders and disease severity exists in these families.

The present study had three aims: first, to examine whether BD-I and BD-II patients from multiplex families with a high density of $\mathrm{BD}$ differ regarding their clinical course, the symptoms presenting during episodes, and psychiatric comorbidities. Second, to analyze whether the genetic risk burden for $\mathrm{BD}, \mathrm{SCZ}$, and MDD differed between the subtypes. Third, to investigate whether the PRS for BD, SCZ, and MDD were higher in patients showing more severe symptoms.

\section{Materials and methods \\ Sample description}

The study subjects are part of the Andalusian Bipolar Family (ABiF) study, which gathered data from 100 Spanish families with at least two cases of BD per family and has been described elsewhere ${ }^{29}$. In the present analyses, $327 \mathrm{BD}$ patients from 98 families were included (BD-I $n=252$, BDII $n=75)$. The individual families were unrelated to each other, and the average pedigree contained 11.8 family members $(\mathrm{SD}=7.6)$, including $3.3(\mathrm{SD}=2.5) \mathrm{BD}$ and 2.0 $(\mathrm{SD}=2.2)$ MDD patients (Supplementary Table S1). Each family contained at least two BD patients. In addition, two pedigrees contained one schizophrenia patient each. The sample included $58.4 \%$ females and had an average age of 48.21 years $(\mathrm{SD}=17.22$; range $=18-96$; age refers to the age either at the interview or at the reported time of the patient's death). The predominant level of education was primary school or less $(70.6 \%)$ and was lower in patients with an earlier decade of birth. Diagnosis and clinical data were based on the Schedule for Affective Disorders and Schizophrenia (SADS) ${ }^{30}$, the Structured Clinical Interview for DSM IV Axis I Disorders (SCID-I) ${ }^{31}$, the Family Informant Schedule and Criteria (FISC) $)^{32}$, and on clinical records. The protocol of the structured diagnostic interview was modified to assess symptoms during lifetime episodes and not only those present in the most severe episode. Diagnoses were given by two trained clinicians using the best estimate approach. For 46 BD-I and 2 BD-II patients, no interview was available, and diagnoses were based on data assessed through best informants only. The local ethics committees of five Andalusian provinces approved the study (Comités de ética de la investigación provincial de Cádiz, Córdoba, Granada, Jaén, and Málaga), and all participants gave their written informed consent.

\section{Genotyping and calculation of PRSs}

Genetic information was available for 156 individuals (BD-I $n=115$, BD-II $n=41$ ) from 33 families. Genomewide genotyping was carried out using the Illumina Infinium PsychArray BeadChip (PsychChip). All quality 
Table 1 Sociodemographic characteristics of BD-I $(n=252)$ and BD-II $(n=75)$ patients.

\begin{tabular}{|c|c|c|c|c|c|c|c|}
\hline Variable & BD-I & $N$ & BD-II & $N$ & $P$ & OR & $95 \% \mathrm{Cl}$ \\
\hline Age & $48(14)$ & 245 & $40(13)$ & 75 & 0.024 & 1.02 & $1.00-1.04$ \\
\hline Gender (female) & $144(57.14)$ & 252 & $47(62.67)$ & 75 & 0.49 & 0.80 & $0.43-1.50$ \\
\hline $\begin{array}{l}\text { Marital status (separated, divorced, or single) } \\
\text { Reference: married or widowed }\end{array}$ & $73(29.08)$ & 251 & 17 (22.67) & 75 & 0.31 & 1.40 & $0.73-2.69$ \\
\hline $\begin{array}{l}\text { Educational level (secondary school or university degree) } \\
\text { Reference: Primary or }<4 \text { years of school }\end{array}$ & $66(26.19)$ & 252 & 29 (39.19) & 74 & 0.38 & 0.74 & $0.38-1.45$ \\
\hline
\end{tabular}

$B D$ bipolar disorder, $n$ valid sample size for the variable, $O R$ odds ratio, $95 \% C l 95 \%$ confidence interval. Bonferroni-corrected threshold for significance: $\alpha=0.05 / 37=$ $1.35 \times 10^{-03}$.

Age refers to the age either at the interview or at the reported time of the patient's death. Age and gender were used as independent variables, with BD type as the dependent variable. Marital status and educational levels were used as dependent variables, with BD type as the independent variable. We used the following covariates: marital status, sex; educational level, sex and decade of birth. The age is described by median and median absolute deviation, sample size ( $N$ ) and percentage are provided for categorical variables.

control (QC) and imputation procedures have been described previously ${ }^{28}$. In brief, QC and population substructure analyses were performed in PLINK v1.9 ${ }^{33}$, as described in the Supplementary Methods. The data were imputed to the 1000 Genomes phase 3 reference panel using SHAPEIT and IMPUTE2 ${ }^{34-36}$. The imputed dataset contained 8,628,089 variants.

For the calculation of $\mathrm{PRSs}^{37}$, SNP weights were estimated using the PRS-CS method ${ }^{38}$ with default parameters (see the Supplementary Methods). This method employs Bayesian regression to infer PRS weights while modeling the local linkage disequilibrium patterns of all SNPs using the EUR super-population of the 1000 Genomes reference panel, without requiring the calculation of several PRS using different $p$-value thresholds. The global shrinkage parameter $\phi$ was determined automatically (PRS-CS-auto; BD: $\phi=1.22 \times 10^{-4}$, MDD: $\phi=1.26 \times$ $10^{-4}$, BD: $\phi=1.47 \times 10^{-4}$ ). The PRSs were calculated, using these weights, in PLINK v1.90b6.13 on imputed dosage data ${ }^{33}$. As training data, we used summary statistics of genome-wide association studies (GWAS) by the Psychiatric Genomics Consortium (PGC) containing 20,352 cases and 31,358 controls for $\mathrm{BD}^{19}, 170,756$ cases and 329,443 controls for $\mathrm{MDD}^{39}$, and 33,640 cases and 43,456 controls for $\mathrm{SCZ}^{40}$. As the ABiF cohort was part of the PGC BD GWAS ${ }^{19}$, Spanish samples were excluded from the BD training GWAS to avoid bias caused by sample overlap.

\section{Generalized estimating equations}

We used generalized estimating equations (GEEs), calculated using the package geepack ${ }^{41}$ in $R$ v4.0.2, to analyze whether BD-I and BD-II differed regarding their demographic information, clinical course, symptoms presenting during episodes, and psychiatric comorbidities. GEEs are suitable for correlated data in samples with a family structure. An exchangeable correlation structure was selected, as is appropriate for the family structure of the sample. To allow for a stable fit, models were only calculated for variables that contained $\geq 10$ individuals within each category (e.g., symptoms present and not present). For variables that did not meet this requirement, no test coefficients are provided in Tables 1-4. Secondary analyses including only probands with a personal interview are provided in Supplementary Table S2.

Sex and age were used as covariates in all analyses of non-sociodemographic, dichotomous variables. Only sex was used for quantitative clinical variables that already contained a temporal component. Quantitative variables were transformed using inverse rank-based normal transformation. The number of episodes and suicide attempts were divided by the illness duration before the transformation. Here, the illness duration is defined as the difference between the age and the age at $\mathrm{BD}$ onset. This ratio allowed for a better normalization than was observed when transforming the variables by themselves and adding the illness duration as a covariate to the model. Secondary analyses were conducted with illness duration as a covariate instead of dividing the numbers by the duration (Supplementary Table S3).

\section{Correction for multiple testing}

To establish an appropriate correction threshold for multiple comparisons using Bonferroni's method, the number of independent tests reported in Tables 1-4 was estimated using principal component analysis (PCA) in $R$ using the function prcomp. Of the 42 variables analyzed, the first 37 components jointly explained $>99 \%$ (99.3\%) of the phenotypic variance (Supplementary Fig. S1). We thus set the significance threshold to $\alpha=0.05 / 37=1.35 \times$ $10^{-03}$. We used a more liberal threshold to select variables for genetic analyses: the first nine components explained $>50 \%(53.1 \%)$ of the phenotypic variance, corresponding to a significance threshold of $\alpha=0.05 / 9=5.56 \times 10^{-03}$. 
Table 2 Differences in the clinical course between BD-I and BD-II patients.

\begin{tabular}{|c|c|c|c|c|c|c|c|}
\hline Variable & BD-I & $N$ & BD-II & $N$ & $P$ & $\beta$ & SE \\
\hline Age at first episode (years) & $21(5)$ & 233 & $21(6)$ & 75 & 0.52 & 0.10 & 0.15 \\
\hline Age at first manic episode (years) & $25(7)$ & 230 & $26(8)$ & 70 & 0.75 & 0.05 & 0.14 \\
\hline Age at first depressive episode (years) & $22(5.5)$ & 230 & $21(6)$ & 75 & 0.59 & 0.08 & 0.16 \\
\hline Duration of illness (years) & $23(11)$ & 233 & $16(9)$ & 75 & $2.83 \times 10^{-03}$ & 0.36 & 0.12 \\
\hline Duration of depressive episodes (weeks) & $16(8)$ & 247 & $12(8)$ & 75 & 0.14 & 0.21 & 0.14 \\
\hline Number of depressive episodes/illness duration & $0.86(0.57)$ & 229 & $0.78(0.58)$ & 75 & 0.27 & 0.17 & 0.16 \\
\hline Number of (hypo)manic episodes/illness duration & $0.52(0.38)$ & 232 & $0.77(0.65)$ & 75 & 0.029 & -0.35 & 0.16 \\
\hline Number of suicide attempts/illness duration (median) & $0(0)$ & 233 & $0(0)$ & 75 & 0.046 & 0.20 & 0.10 \\
\hline Number of suicide attempts/illness duration (mean) & $0.04(0.14)$ & 233 & $0.02(0.08)$ & 75 & 0.046 & 0.20 & 0.10 \\
\hline Variable & BD-I & $N$ & BD-II & $N$ & $P$ & OR & $95 \% \mathrm{Cl}$ \\
\hline Depressive polarity of the first episode & $102(43.78)$ & 233 & $38(50.7)$ & 75 & 0.22 & 0.75 & $0.48-1.18$ \\
\hline ECT during depressive episodes & $9(3.64)$ & 247 & $1(1.33)$ & 75 & 0.42 & 2.41 & $0.28-20.95$ \\
\hline Medication during depressive episodes & $237(95.18)$ & 249 & $61(81.33)$ & 75 & 0.010 & 3.93 & $1.38-11.20$ \\
\hline Hospitalization during depressive episodes & $50(20.16)$ & 248 & $3(4.00)$ & 75 & $2.45 \times 10^{-03}$ & 6.41 & $1.93-21.35$ \\
\hline Incapacity during depressive episodes & $158(69.30)$ & 228 & $33(45.83)$ & 72 & $7.07 \times 10^{-04}$ & 2.51 & $1.47-4.28$ \\
\hline Suicide attempted (ever) & $65(25.79)$ & 252 & $11(14.67)$ & 75 & 0.040 & 2.46 & $1.04-5.79$ \\
\hline Serious or extreme suicide attempt & $18(7.14)$ & 252 & $2(2.66)$ & 75 & 0.13 & 3.44 & $0.70-17.03$ \\
\hline Seasonality & $139(58.65)$ & 237 & $36(49.31)$ & 73 & 0.043 & 1.73 & $1.02-2.95$ \\
\hline
\end{tabular}

$B D$ bipolar disorder, $S E$ standard error, $N$ sample size for the variable (before correction for covariates), $O R$ odds ratio, $95 \%$ CI $95 \%$ confidence interval, $E C T$ electroconvulsive therapy. Bonferroni-corrected threshold for significance: $a=0.05 / 37=1.35 \times 10^{-03}$.

Quantitative variables were analyzed in linear, dichotomous variables in logistic models. BD type was the independent variable in all models. We used the following covariates: quantitative variables, sex; dichotomous variables, sex and age. All quantitative variables have been transformed for the analyses using inverse rank-based normalization to generate normally distributed residuals. The numbers of episodes and suicide attempts were divided by the illness duration before the transformation. See Supplementary Table S6 for analyses of these variables not divided by illness duration. All quantitative variables are described by median and median absolute deviation of untransformed variables, sample size $(N)$ and percentage are provided for categorical variables. For the number of suicide attempts, mean and standard deviation are also provided. Significant variables are labeled in bold font.

\section{Analyses of PRSs}

PRS were analyzed to compare the polygenic risk burden for $\mathrm{BD}, \mathrm{MDD}$, and SCZ between $\mathrm{BD}$ types and to explore the influence of PRS on the clinical characteristics. We analyzed the association of PRS with all dichotomous phenotypes showing a $p<5.56 \times 10^{-03}$ in GEE analyses (see above). PRS analyses were carried out as previously described ${ }^{28}$ in $R$ using the function $\mathrm{glmm}$. wald of the package GMMAT $T^{42}$, fitted by maximum likelihood using Nelder-Mead optimization. Family structure was modeled as a random effect, with a genetic relationship matrix calculated on pruned genotype data using GEMMA ${ }^{43}$.

PRSs were transformed by Z-score standardization before the analyses. Age and sex were used as fixedeffects covariates in all analyses. We used Bonferroni's method to correct for three tests $(\alpha=0.05 / 3=0.0167)$ in the analyses of PRS with BD type and for 18 tests (three PRS and six variables) in the analyses of PRS with clinical course and symptoms variables $(\alpha=0.05$ / $\left.18=2.78 \times 10^{-03}\right)$. Based on a previous report ${ }^{19}$, we expected higher SCZ PRS in BD-I cases and higher MDD PRS in BD-II cases. Here, also one-sided $p$-values are reported.

\section{Permutation analyses}

All association $p$-values were validated using permutation analyses. Here, the null distribution of test statistics was empirically determined by repeating regression analyses with a random sampling of phenotype data. To calculate a $p$-value, the number of tests were counted where a model with a random association showed the same or a more extreme, i.e., smaller $p$-value than the correct, non-randomized model; this number was divided by the total number of tests. Results from these permutation analyses are, together with the respective number of permutations, presented in Supplementary Tables S3S6. Some permutation $p$-values were higher than the 
Table 3 Comparison of the symptoms profiles during lifetime between BD-I and BD-II patients.

\begin{tabular}{|c|c|c|c|c|c|c|c|c|}
\hline Symptoms during manic/hypomanic episodes & BD-I & & $N$ & BD-II & $N$ & $P$ & OR & $95 \% \mathrm{Cl}$ \\
\hline Hyperactivity & $252(100)$ & & 252 & $75(100)$ & 75 & & & \\
\hline Talkativeness & $252(100)$ & & 252 & $73(97.33)$ & 75 & & & \\
\hline Flight of idea & $236(99.58)$ & & 237 & $68(97.14)$ & 70 & & & \\
\hline Inflated grandiosity & $248(99.20)$ & & 250 & $73(97.33)$ & 75 & & & \\
\hline Decreased need for sleep & $251(99.60)$ & & 252 & $72(96.00)$ & 75 & & & \\
\hline Inattention & $239(95.60)$ & & 250 & $64(85.33)$ & 75 & $7.19 \times 10^{-04}$ & 4.79 & $1.93-11.88$ \\
\hline Reckless behavior & $166(66.13)$ & & 251 & $9(12.16)$ & 73 & $3.95 \times 10^{-13}$ & 13.48 & $6.68-27.20$ \\
\hline Delusions & $217(87.85)$ & & 247 & 0 & 75 & & & \\
\hline Hallucinations & $20(8.16)$ & & 245 & 0 & 75 & & & \\
\hline Symptoms during depressive episodes & BD-I & $N$ & & BD-II & $N$ & $P$ & OR & $95 \% \mathrm{Cl}$ \\
\hline Appetite change & $240(96.38)$ & 249 & & $70(93.33)$ & 75 & 0.25 & 1.80 & $0.66-4.94$ \\
\hline Loss of appetite or weight & $209(90.87)$ & 230 & & $67(93.05)$ & 72 & 0.61 & 0.76 & $0.27-2.15$ \\
\hline Increased appetite or weight & $15(6.52)$ & 230 & & $5(6.94)$ & 72 & 0.91 & 1.05 & $0.46-2.37$ \\
\hline Sleep change & $245(98.39)$ & 249 & & $73(97.33)$ & 75 & & & \\
\hline Early morning awakening & $176(79.64)$ & 221 & & $48(67.60)$ & 71 & 0.030 & 1.82 & $1.06-3.11$ \\
\hline Hypersomnia & $5(2.16)$ & 231 & & $2(2.78)$ & 72 & & & \\
\hline Psychomotor alterations & $243(97.59)$ & 249 & & $72(96.00)$ & 75 & & & \\
\hline Fatigue or tiredness & $248(99.60)$ & 249 & & $74(98.67)$ & 75 & & & \\
\hline Diminished libido & $236(97.52)$ & 242 & & $71(94.96)$ & 74 & & & \\
\hline Guilt & $215(86.34)$ & 249 & & $63(84.00)$ & 75 & 0.97 & 1.02 & $0.36-2.91$ \\
\hline Difficult thinking or indecisiveness & $241(96.79)$ & 249 & & $72(96.00)$ & 75 & 0.15 & 2.18 & $0.75-6.34$ \\
\hline Suicidal ideation & $222(89.15)$ & 249 & & $57(72.00)$ & 75 & $4.09 \times 10^{-03}$ & 2.51 & $1.34-4.70$ \\
\hline Loss of pleasure & $221(95.26)$ & 232 & & $69(95.83)$ & 72 & 0.98 & 1.02 & $0.24-4.36$ \\
\hline Lack of reactivity & $149(64.78)$ & 230 & & $44(61.11)$ & 72 & 0.81 & 1.07 & $0.63-1.80$ \\
\hline Different feeling of sadness & 206 (94.49) & 218 & & $68(94.44)$ & 72 & 0.88 & 1.10 & $0.32-3.72$ \\
\hline Morning worsening & $175(76.75)$ & 228 & & $46(63.89)$ & 72 & 0.025 & 1.86 & $1.08-3.21$ \\
\hline Excessive guilt & $196(84.48)$ & 232 & & $59(81.94)$ & 72 & 0.65 & 1.24 & $0.49-3.13$ \\
\hline Leaden paralysis & $103(44.59)$ & 231 & & $24(33.33)$ & 72 & 0.05 & 1.81 & $1.00-3.27$ \\
\hline Delusions & $35(14.11)$ & 248 & & $3(4.00)$ & 75 & $3.56 \times 10^{-03}$ & 4.80 & $1.67-13.78$ \\
\hline Hallucinations & $28(11.29)$ & 248 & & $2(2.67)$ & 75 & 0.021 & 5.57 & $1.29-24.04$ \\
\hline
\end{tabular}

$B D$ bipolar disorder, $n$ valid sample size for the variable, OR odds ratio, $95 \%$ Cl $95 \%$ confidence interval. Bonferroni-corrected threshold for significance: $\alpha=0.05 / 37=$ $1.35 \times 10^{-03}$

BD type was the independent variable in all models. Sample size $(N)$ and percentage are provided for categorical variables. Significant variables are labeled in bold font. To allow for a stable fit, models were only calculated for variables that contained $\geq 10$ individuals per variable category. Variables without test coefficients $(P, O R$, $\mathrm{Cl})$ did not meet this requirement.

$p$-values directly estimated with GEE models, but all variables that were significant in the primary GEE analyses showed a permutation $p \leq 2.66 \times 10^{-03}$ (Supplementary Table S4). Typically, the permutation $p$-values of PRS analyses were lower than the $p$-values from GMMAT Wald tests (Supplementary Tables S5-S6).

\section{Power analysis}

We conducted power analyses for classical, $p$-value threshold-based PRS using AVENGEME ${ }^{44}$. Because PRS calculated by PRS-CS have a higher power ${ }^{45}$, we assume that these estimates constitute lower boundaries of our real statistical power. According to these analyses, we had a power 
Table 4 Comparison of comorbidities between BD-I and BD-II patients.

\begin{tabular}{|c|c|c|c|c|c|c|c|}
\hline Comorbidity & BD-I & $N$ & BD-II & $N$ & $P$ & OR & $95 \% \mathrm{Cl}$ \\
\hline Alcohol abuse & $30(11.90)$ & 252 & $5(6.67)$ & 75 & 0.32 & 1.72 & $0.59-5.00$ \\
\hline Drug abuse & $15(5.95)$ & 252 & $6(8.00)$ & 75 & 0.87 & 1.09 & $0.38-3.13$ \\
\hline Alcohol dependence & $7(2.78)$ & 252 & $3(4.00)$ & 75 & 0.46 & 0.65 & $0.20-2.07$ \\
\hline Drug dependence & $3(1.19)$ & 252 & 0 & 75 & & & \\
\hline Panic disorder & $6(2.38)$ & 252 & 0 & 75 & & & \\
\hline Obsessive compulsive disorder & $2(0.80)$ & 251 & 0 & 75 & & & \\
\hline Phobia & $2(0.80)$ & 251 & 0 & 75 & & & \\
\hline Somatoform disorder & $2(0.83)$ & 242 & 0 & 71 & & & \\
\hline Cyclothymic personality & $26(10.32)$ & 252 & $7(9.33)$ & 75 & 0.97 & 1.02 & $0.43-2.41$ \\
\hline Any comorbid disorder & $71(28.17)$ & 252 & $17(22.67)$ & 75 & 0.31 & 1.38 & $0.74-2.57$ \\
\hline
\end{tabular}

$B D$ bipolar disorder, $N$ valid sample size for the variable, $O R$ odds ratio, $95 \%$ Cl $95 \%$ confidence interval. Bonferroni-corrected threshold for significance: $a=0.05 / 37=$ $1.35 \times 10^{-03}$

BD type was the independent variable in all models. Sample size $(N)$ and percentage are provided for categorical variables. To allow for a stable fit, models were only calculated for variables that contained $\geq 10$ individuals per variable category. Variables without test coefficients $(\mathrm{P}, \mathrm{OR}, \mathrm{Cl})$ did not meet this requirement.

of 0.64, 0.73, and 0.39 for analyses of BD-I vs. BD-II with PRS for BD, SCZ, and MDD, respectively (Supplementary Table S7). For the quantitative traits, the power was 0.91, 0.95 , and 0.65 for $\mathrm{BD}, \mathrm{SCZ}$, and $\mathrm{MDD}$, respectively.

\section{Results}

The sociodemographic variables did not differ significantly between family members diagnosed with BD-I and BD-II (Table 1). Among the variables related to the clinical course of disorder (Table 2), BD-I patients more frequently showed incapacity during depressive episodes (odds ratio $(\mathrm{OR})=2.51,95 \%$ confidence interval $(\mathrm{CI})=$ 1.47-4.28, $\left.p=7.07 \times 10^{-04}\right)$ after Bonferroni correction for 37 independent tests $\left(\alpha=1.35 \times 10^{-03}\right.$; 37 principal components explain $99.3 \%$ of the phenotypic variance). Furthermore, BD-I patients showed a longer average illness duration $(\beta=0.36$ standard deviations, $\mathrm{SE}=0.12$, $p=2.83 \times 10^{-03}$ ) and more hospitalizations during depressive episodes $(\mathrm{OR}=6.41, \mathrm{CI}=1.93-21.35, \quad p=$ $\left.2.45 \times 10^{-03}\right)$, yet those two analyses did not withstand correction for multiple testing.

During (hypo)manic episodes, BD-I patients exhibited significantly more inattention $(\mathrm{OR}=4.79, \quad \mathrm{CI}=$ 1.93-11.88, $p=7.19 \times 10^{-04}$; Table 3) and reckless behavior $\left(\mathrm{OR}=13.48, \mathrm{CI}=6.68-27.20, p=3.95 \times 10^{-13}\right)$. The $p$-value of reckless behavior was higher in the permutation analysis but remained significant $(p=1.26 \times$ $10^{-04}$, Supplementary Table S4). During depressive episodes, suicidal ideation $(\mathrm{OR}=2.51, \mathrm{CI}=1.34-4.70, p=$ $\left.4.09 \times 10^{-03}\right)$ and delusions $(\mathrm{OR}=4.80, \mathrm{CI}=1.67-13.78$, $p=3.56 \times 10^{-03}$ ) were observed more frequently in BD-I patients, but these associations were not significant after correction for multiple testing. BD-I and BD-II patients did not differ significantly regarding the examined comorbidities with other mental disorders (Table 4).

When only analyzing patients with a personal interview, incapacity during depressive episodes and reckless behavior remained significant (Supplementary Table S2). In these secondary analyses, inattention did not remain significant after correction for multiple testing. By contrast, medication and hospitalization during depressive episodes, both only nominally significant in the primary analysis, were significant after correction for multiple testing in the secondary analysis.

Next, we analyzed PRS in 156 of the 327 patients for which genetic data were available. In this smaller subset, the statistical power was reduced compared to the phenotype-level analyses (Supplementary Table S7). In our analysis whether PRS for $\mathrm{BD}^{19}, \mathrm{MDD}^{39}$, or $\mathrm{SCZ}^{40}$ differed between BD subtypes (Fig. 1A, Supplementary Table S4), BD-II cases showed a significantly higher MDD risk burden after correction for three tests $(\mathrm{OR}=1.70$, $\mathrm{CI}=1.14-2.53, \quad p=9.11 \times 10^{-03}, \quad p_{\text {one-sided }}=4.55 \times$ $\left.10^{-03}\right)$. No significant differences were observed for $\mathrm{BD}$ and SCZ PRS.

We finally analyzed whether PRS were higher in patients showing a more severe clinical course or more severe symptoms. We conducted these analyses for the six dichotomous variables differing between BD subtypes in primary analyses, prioritized using a $p$-value threshold of $\alpha=5.56 \times 10^{-03}$ (nine components explain $53.1 \%$ of the phenotypic variance). BD PRS were significantly higher in patients showing suicidal ideation after Bonferroni correction for 18 tests $(\mathrm{OR}=2.25, \mathrm{CI}=1.38-3.67, p=1.11 \times$ $10^{-03}, p_{1 \text {-sided }}=5.53 \times 10^{-04}$, Fig. $1 \mathrm{~B}$, Supplementary Table S5). Furthermore, BD PRS were higher in patients 

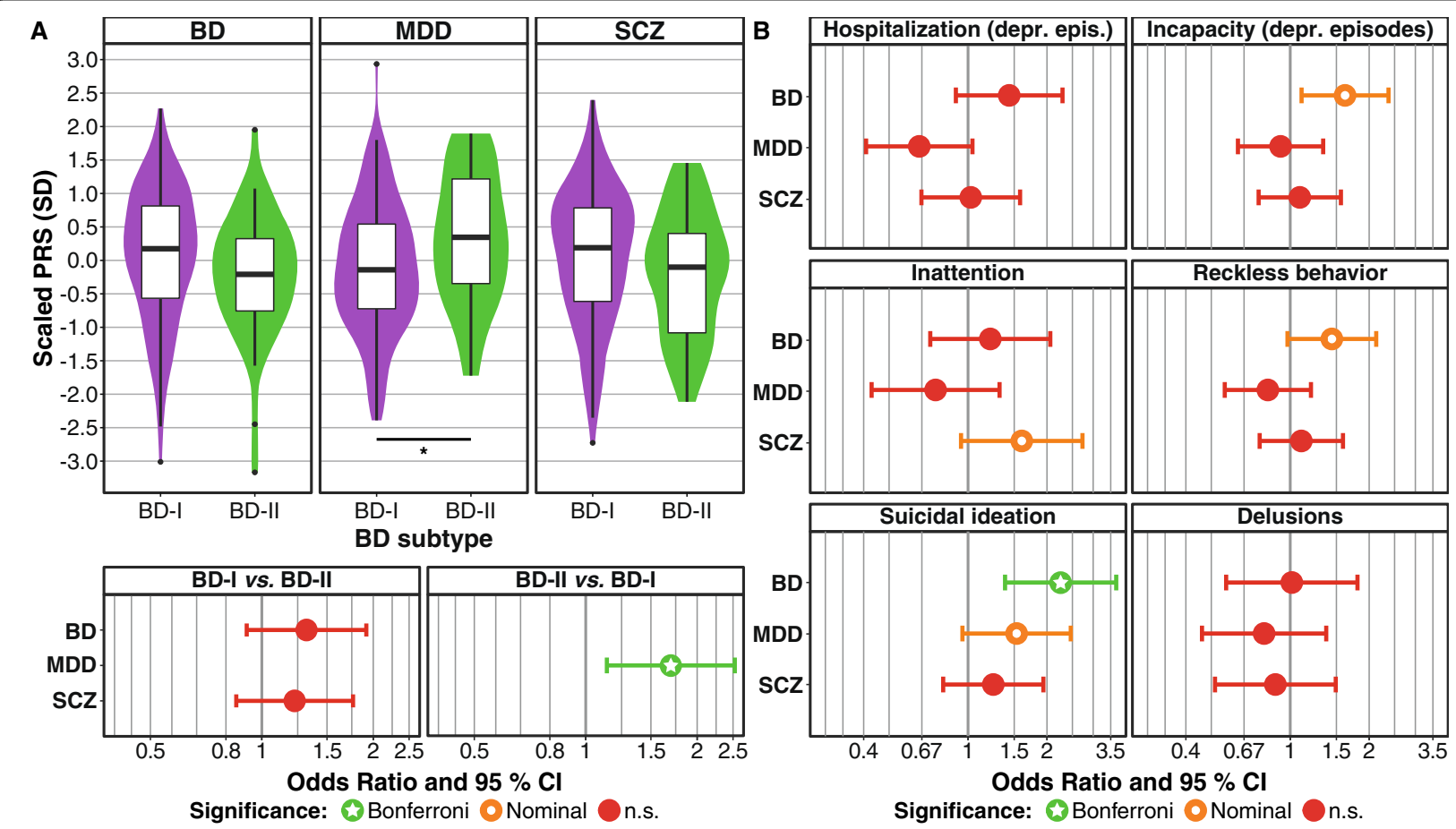

Fig. 1 Polygenic risk score analyses in a subset of 156 family members. A BD-II patients showed a significantly higher MDD PRS than BD-I cases. SD standard deviation. Results from a logistic mixed regression model using PRS as predictors, BD type as the outcome, and sex and age as covariates (for full results see Supplementary Table S5). Comparisons significant after Bonferroni correction for three tests $(a=0.05 / 3=0.0167)$ are marked with an asterisk. For the SCZ PRS, the one-sided hypothesis was that BD-I cases have higher PRS; for the MDD PRS, the one-sided hypothesis was that BD-II cases show higher PRS. B Analysis of dichotomous clinical course and symptom variables with $p<5.56 \times 10^{-03}$ in the phenotypic analyses (see Tables 2-3). Results from a logistic mixed regression model using PRS as predictors, symptoms as the outcome, and sex and age as covariates (for full results see Supplementary Table S6). Bonferroni-corrected threshold for significance: $a=0.05 /(6 \times 3)=2.78 \times 10^{-03}$. For all PRS, the one-sided hypothesis was that the symptom severity increases with the PRS. PRS polygenic risk score, BD bipolar disorder, MDD major depressive disorder, SCZ schizophrenia, 95\% Cl 95\% confidence interval, depr. epis. depressive episodes.

with incapacity during depressive episodes, yet this association did not remain significant after correction for multiple testing.

\section{Discussion}

The present study investigated phenotypic and genetic differences between BD-I and BD-II patients of BD multiplex families: First, patients diagnosed with BD-I showed both more severe manic episodes, with frequent inattention and reckless behavior, and more serious depressive episodes, which were often characterized by incapacity. Second, BD-II patients exhibited a significantly higher genetic MDD risk load than family members diagnosed with BD-I. Third, the genetic risk burden for BD was significantly associated with suicidal ideation.

It is not surprising that we found reckless behavior to be more frequent in BD-I than in BD-II cases, as it constitutes one of the main reasons for hospitalization. Previous studies have already described reckless behavior and inattention as features of BD-1 ${ }^{14,46}$.

An increased incapacity of BD-I patients during depressive episodes was previously reported in a study conducted in unrelated BD patients ${ }^{7}$. Besides increased occupational impairment, further severity-related measures were described in this previous study, e.g., an increased need for medication and psychiatric hospitalizations, both of which only achieved nominal significance in the present study but were significant in our secondary analyses of patients with a personal interview. Furthermore, a tendency of BD-I patients to display more psychotic symptoms during their depressive episodes did not remain significant after correction for multiple testing in the present study. Previous findings regarding differences in the number of depressive episodes between BD-I and BD-II patients-not significant in the present study-were mixed: Studies in multiplex families found either no difference $^{47}$ or more depressive episodes for BD-I ${ }^{26}$. Conversely, studies in unrelated patients in a clinical setting found more depressive episodes in BD-II patients ${ }^{12,13}$.

Berskon's selection bias might have contributed to the observation of an increased severity and a higher number of depressive episodes in unrelated BD-II patients recruited from a clinical setting: It seems plausible that clinical samples of sporadic BD-II patients are enriched 
for patients suffering from more and more severe depressive episodes, for which they sought clinical help. Findings from the World Mental Health Survey Initiative (cross-sectional face-to-face interviews in 61,392 community-based adults from eleven countries) ${ }^{1}$ support this idea, finding that the severity of manic and depressive symptoms and of suicidal behavior increased monotonically from subthreshold BD to BD-II to BD-I.

Suicidal ideation was nominally associated with BD-I and can be considered a highly relevant indicator of mental disorder severity. Prior findings on the difference of suicidal ideations between BD-I and BD-II in samples of unrelated patients were inconsistent ${ }^{3,7,14}$. A large published epidemiological study investigating differences in suicidal ideation between the two subtypes in more than $1400 \mathrm{BD}$ patients, found more suicidal ideation during depressive episodes in BD-I cases ${ }^{7}$. However, this difference was not observed in a previous study of multiplex families ${ }^{26}$. Published results are also mixed regarding lifetime suicide attempts ${ }^{8,14,48-52}$. A metaanalysis indicated possibly more suicide attempts in BDI cases $^{53}$, but this result was not significant $(p=0.07)$. Although suicide attempts occurred more frequently in BD-I than BD-II patients in the ABiF sample, this difference did not reach significance in the present study.

We have described previously that both affected and unaffected $\mathrm{ABiF}$ family members exhibit increased genetic risk loads for $\mathrm{BD}, \mathrm{MDD}$, and $\mathrm{SCZ}$, compared to unaffected controls ${ }^{28}$. Within the families, BD patients showed higher BD PRS than unaffected family members ${ }^{28}$. In the present study, BD-II patients had a higher MDD risk load than BD-I patients, consistent with previous results from unrelated cases ${ }^{19}$. We observed a tendency for higher BD and SCZ PRS in BD-I patients, but both differences were not significant, possibly due to the small sample size, which impeded conclusive interpretations.

These findings do not unequivocally support the hypothesis that the BD genetic risk load shapes the development of either BD-II or BD-I along a severity continuum, i.e., that BDI cases carry more BD risk variants than BD-II cases. Instead, our previous ${ }^{28}$ and present results could suggest that, in the ABiF families, a general load of psychiatric, and especially $\mathrm{BD}$, risk variants drive the overall $\mathrm{BD}$ vulnerability, while an increased MDD risk load may have shaped the family members' BD presentation towards the BD-II subtype. The association of the BD PRS with suicidal ideation indicates that the polygenic makeup might, beyond its contribution to categorical subtypes of $\mathrm{BD}$, shape the patients' individual disorder manifestation on a symptom level. However, more studies with larger samples are needed to confirm these hypotheses.

There were several limitations of this study. We analyzed lifetime symptoms and not only symptoms during the worst episode, which should be kept in mind when interpreting and comparing the results to other studies. Furthermore, the small sample size, especially in the genetic analyses, limits the generalizability of results obtained and increases the likelihood of type II errors. Thus, these results need to be interpreted with caution. However, the family-based design and the homogeneity of the sample may have improved statistical power. Still, future studies in the ABiF study and other family cohorts should aim to analyze larger samples. Moreover, the present study only analyzed common genetic variants, and rare variants could also have contributed to clinical differences of BD patients in the ABiF multiplex families ${ }^{54,55}$. Accordingly, a notable disadvantage of studying multiplex families is that they may harbor more rare variants than patients recruited from the general population. In addition, their common variant patterns and shared environmental factors may differ from those observed in unrelated case/control samples. We analyzed a multi-generational study, and demographic factors, especially the educational system and the access to it, have changed during the $20^{\text {th }}$ century in Spain. In earlier generations, the average educational level was lower, and more family members were married. The GWAS used for calculating the BD PRS contained over four times as many BD-I than BD-II cases ${ }^{19}$ and, therefore, the BD PRS was likely more sensitive for the genetic architecture of BD-I than for BD-II.

In summary, the present study compared clinical, symptomatological, and genetic features of BD-I and BD-II patients from $\mathrm{BD}$ multiplex pedigrees. The finding that $\mathrm{BD}$ I patients showed more severe symptoms during their manic as well as during their depressive episodes, and the association of the BD PRS with suicidal ideation, are in line with a genetic severity continuum in multiplex BD families. The observation of a lower genetic risk load for MDD in BD-I patients, however, points to a more complex situation: It indicates that the individual polygenic risk load for different psychiatric disorders may influence the development of either BD-I or BD-II and the associated symptoms. Future studies should aim to replicate these results and examine the underlying mechanisms, e.g., biological pathways or potentially protective effects of high MDD PRS on the development of pronounced manic symptoms.

\footnotetext{
Acknowledgements

The study was supported by the German Federal Ministry of Education and Research (BMBF) through the Integrated Network IntegraMent under the auspices of the e:Med programme (grants 01ZX1314A to MMN and SC; 01ZX1314G and 01ZX1614G to MR), and through "AERIAL - Addiction: Early Recognition and Intervention Across the Lifespan" (grant 01EE1406C to MR), through "ASD-Net" (grant 01EE1409C to MR and SHW); by ERA-NET NEURON through "SynSchiz - Linking synaptic dysfunction to disease mechanisms in schizophrenia_a multilevel investigation" (grant 01EW1810 to MR) and "EMBED" (grant 01EW1904); by the German Research Foundation (DFG) through "FOR2107" (grants RI908/11-2 to MR; NO246/10-2 to MMN and WI3439/3-2 to SHW); by the Andalusian regional Health and Innovation Government (grants PI-0060-2017, RC-0006-2015, and the Nicolas Monardes Programme to YDO (CTS-546)); and by the Swiss National Science Foundation
} 
(156791 to SC). MMN is a member of the DFG-funded cluster of excellence ImmunoSensation. TFMA was supported by the BMBF through the DIFUTURE consortium of the Medical Informatics Initiative Germany (grant 01ZZ1804A) and the European Union's Horizon 2020 Research and Innovation Programme (grant MultipleMS, EU RIA 733161).

\section{Author details}

${ }^{1}$ Department of Mental Health, University Regional Hospital of Málaga, Institute of Biomedicine of Málaga (IBIMA), Málaga, Spain. ${ }^{2}$ Department of Genetic Epidemiology in Psychiatry, Central Institute of Mental Health, Medical Faculty Mannheim, Heidelberg University, Mannheim, Germany. ${ }^{3}$ Centre for Human Genetics, University of Marburg, Marburg, Germany. ${ }^{4}$ Institute of Human Genetics, University of Bonn, School of Medicine \& University Hospital Bonn, Bonn, Germany. ${ }^{5}$ Department of Mental Health, Hospital of Puerto Real, Cádiz, Spain. ${ }^{6}$ Department of Mental Health, University Hospital of Reina Sofia, Cordoba, Spain. ${ }^{7}$ Department of Mental Health, Hospital of Jaen, Jaen, Spain. ${ }^{8}$ Department of Mental Health, Hospital of Jerez de la Frontera, Jerez de la Frontera, Spain. 'Department of Mental Health, Hospital Punta de Europa, Algeciras, Spain. ${ }^{10}$ Unidad de Gestión Clínica del Dispositivo de Cuidados Críticos y Urgencias del Distrito Sanitario Málaga-Coin-Guadalhorce, Málaga, Spain. ${ }^{11}$ Department of Personality, Assessment and Psychological Treatment, University of Malaga, Institute of Biomedicine of Málaga (IBIMA), Málaga, Spain. ${ }^{12}$ Department of Neurology, Goethe University Medical School, Frankfurt am Main, Germany. ${ }^{13}$ Department of Biomedicine, University of Basel, Basel, Switzerland. ${ }^{14}$ Institute of Neuroscience and Medicine (INM-1), Research Center Jülich, Jülich, Germany. ${ }^{15}$ Department of Neurology, Klinikum rechts der Isar, School of Medicine, Technical University of Munich, Munich, Germany

\section{Funding}

Open Access funding enabled and organized by Projekt DEAL.

\section{Conflict of interest}

The authors declare that they have no conflict of interest.

\section{Publisher's note}

Springer Nature remains neutral with regard to jurisdictional claims in published maps and institutional affiliations.

Supplementary Information accompanies this paper at (https://doi.org/ 10.1038/s41398-020-01146-0).

Received: 23 October 2020 Revised: 30 November 2020 Accepted: 4 December 2020

Published online: 11 January 2021

\section{References}

1. Merikangas, K. R. et al. Prevalence and correlates of bipolar spectrum disorder in the world mental health survey initiative. Arch. Gen. Psychiatry 68, 241-251 (2011).

2. American Psychiatric Association. Diagnostic and Statistical Manual of Mental Disorders. 5h edn (DSM-5(TM)). (American Psychiatric Publishing, Arlington, VA, 2013).

3. Parker, G. et al. Differentiation of bipolar I and II disorders by examining for differences in severity of manic/hypomanic symptoms and the presence or absence of psychosis during that phase. J. Affect Disord. 150, 941-947 (2013).

4. Brugue, E., Colom, F., Sanchez-Moreno, J., Cruz, N. \& Vieta, E. Depression subtypes in bipolar I and II disorders. Psychopathology 41, 111-114 (2007).

5. Goes, F. S. et al. Psychotic features in bipolar and unipolar depression. Bipolar Disord. 9, 901-906 (2007).

6. Altamura, A. C. et al. Socio-demographic and clinical characterization of patients with Bipolar Disorder I vs II: a Nationwide Italian Study. Eur. Arch. Psychiatry Clin. Neurosci. 268, 169-177 (2018).

7. Bega, S., Schaffer, A., Goldstein, B. \& Levitt, A. Differentiating between bipolar disorder types I and II: Results from the national epidemiologic survey on alcohol and related conditions (NESARC). J. Affect Disord. 138, 46-53 (2012).

8. Karanti A. et al. Characteristics of bipolar I and II disorder: a study of 8766 individuals. Bipolar Disord. https://doi.org/10.1111/bdi.12867 (2019).
9. Cotrena, C. et al. Executive functions and memory in bipolar disorders I and II: new insights from meta-analytic results. Acta Psychiatr. Scand. 141, 110-130 (2020).

10. Vieta, E. \& Suppes, T. Bipolar II disorder: arguments for and against a distinct diagnostic entity. Bipolar Disord. 10, 163-178 (2008).

11. Parker, G. B., Romano, M., Graham, R. K. \& Ricciardi, T. Comparative familial aggregation of bipolar disorder in patients with bipolar I and bipolar II disorders. Australas. Psychiatry 26, 414-416 (2018).

12. Vieta, E., Gastó, C., Otero, A., Nieto, E. \& Vallejo, J. Differential features between bipolar I and bipolar II disorder. Compr. Psychiatry 38, 98-101 (1997).

13. Judd, L. L. et al. The comparative clinical phenotype and long term longitudinal episode course of bipolar I and II: A clinical spectrum or distinct disorders? J. Affect Disord. 73, 19-32 (2003).

14. Baek, J. H. et al. Differences between bipolar i and bipolar II disorders in clinical features, comorbidity, and family history. J. Affect Disord. 131, 59-67 (2011).

15. Vinberg, M., Mikkelsen, R. L., Kirkegaard, T., Christensen, E. M. \& Kessing, L. V. Differences in clinical presentation between bipolar I and II disorders in the early stages of bipolar disorder: a naturalistic study. J. Affect Disord. 208, 521-527 (2017).

16. Albert, U., Rosso, G., Maina, G. \& Bogetto, F. Impact of anxiety disorder comorbidity on quality of life in euthymic bipolar disorder patients: differences between bipolar I and II subtypes. J. Affect Disord. 105, 297-303 (2008).

17. Forte, A. et al. Long-term morbidity in bipolar-l, bipolar-II, and unipolar major depressive disorders. J. Affect Disord. 178, 71-78 (2015).

18. Pallaskorpi, S. et al. Five-year outcome of bipolar I and II disorders: findings of the Jorvi Bipolar Study. Bipolar Disord. 17, 363-374 (2015).

19. Stahl, E. A. et al. Genome-wide association study identifies 30 loci associated with bipolar disorder. Nat. Genet. 51, 793-803 (2019).

20. Song, J. et al. Specificity in etiology of subtypes of bipolar disorder: evidence from a Swedish population-based family study. Biol. Psychiatry 84, 810-816 (2018).

21. Coleman, J. R. I. et al. The genetics of the mood disorder spectrum: genomewide association analyses of more than 185,000 cases and 439,000 controls. Biol. Psychiatry https://doi.org/10.1016/j.biopsych.2019.10.015 (2020).

22. Heun, R. \& Maier, W. The distinction of bipolar II disorder from bipolar I and recurrent unipolar depression: results of a controlled family study. Acta Psychiatr. Scand. 87, 279-284 (1993).

23. Coryell, W., Endicott, J., Reich, T., Andreasen, N. \& Keller, M. A family study of bipolar II disorder. Br. J. Psychiatry 145, 49-54 (1984).

24. Endicott, J. et al. Bipolar II. Combine or keep separate? J. Affect Disord. 8, 17-28 (1985).

25. Ott, J., Kamatani, Y. \& Lathrop, M. Family-based designs for genome-wide association studies. Nat. Rev. Genet 12, 465-474 (2011).

26. Frankland, A. et al. Comparing the phenomenology of depressive episodes in bipolar I and II disorder and major depressive disorder within bipolar disorder pedigrees. J. Clin. Psychiatry 76, 32-39 (2015).

27. Collins, A. L. et al. Identifying bipolar disorder susceptibility loci in a densely affected pedigree. Mol. Psychiatry 18, 1245-1246 (2013).

28. Andlauer, T. F. M. et al. Bipolar multiplex families have an increased burden of common risk variants for psychiatric disorders. Mol. Psychiatry https://doi.org/ 10.1038/s41380-019-0558-2 (2019).

29. Guzman-Parra, J. et al. The Andalusian Bipolar Family (ABiF) Study: protocol and sample description. Rev. Psiquiatr. Salud Ment. 11, 199-207 (2018).

30. Endicott, J. \& Spitzer, R. L. A diagnostic interview: the schedule for affective disorders and schizophrenia. Arch. Gen. Psychiatry 35, 837-844 (1978).

31. First, M. B., Spitzer, R. L., Gibbon, M. \& Williams, J. B. W. Structured clinical interview for Axis I DSM-IV disorders. Patient Ed (SCID-I/P, vs 20) (1994).

32. Mannuzza, S., Fyer, A. J. \& Endicott, J. K. D. Family Informant Schedule and Criteria (FISC) (New York Anxiety Disorders Clinic, New York, 1985).

33. Chang, C. C. et al. Second-generation PLINK: rising to the challenge of larger and richer datasets. Gigascience 4, 7 (2015).

34. Howie, B. N., Donnelly, P. \& Marchini, J. A flexible and accurate genotype imputation method for the next generation of genome-wide association studies. PLoS Genet. 5, e1000529 (2009).

35. Howie, B., Fuchsberger, C., Stephens, M., Marchini, J. \& Abecasis, G. R. Fast and accurate genotype imputation in genome-wide association studies through pre-phasing. Nat. Genet. 44, 955-959 (2012).

36. Delaneau, O., Zagury, J.-F. \& Marchini, J. Improved whole-chromosome phasing for disease and population genetic studies. Nat. Methods 10, 5-6 (2013).

37. Andlauer, M. \& Nöthen, M. M. Polygenic scores for psychiatric disease: from research tool to clinical application. Medizinische Genet. 32, 39-45 (2020). 
38. Ge, T., Chen, C. Y., Ni, Y., Feng, Y. C. A. \& Smoller, J. W. Polygenic prediction via Bayesian regression and continuous shrinkage priors. Nat. Commun. 10, 1-10. (2019).

39. Howard, D. M. et al. Genome-wide meta-analysis of depression identifies 102 independent variants and highlights the importance of the prefrontal brain regions. Nat. Neurosci. 22, 343-352 (2019).

40. Ripke, S. et al. Biological insights from 108 schizophrenia-associated genetic loci. Nature 511, 421-427 (2014).

41. Halekoh, U., Højsgaard, S. \& Yan, J. The R package geepack for generalized estimating equations. J. Stat. Softw. 15, 1-11. (2006).

42. Chen, $\mathrm{H}$. et al. Control for population structure and relatedness for binary traits in genetic association studies via logistic mixed models. Am. J. Hum. Genet. 98 653-666 (2016).

43. Zhou, X. \& Stephens, M. Efficient multivariate linear mixed model algorithms for genome-wide association studies. Nat. Methods 11, 407-409 (2014).

44. Dudbridge, F. Power and predictive accuracy of polygenic risk scores. PLoS Genet. 9, 1003348 (2013).

45. Ni G. et al. A comprehensive evaluation of polygenic score methods across cohorts in psychiatric disorders. MedRxiv https://doi.org/10.1101/ 2020.09.10.20192310 (2020)

46. Serretti, A. \& Olgiati, P. Profiles of 'manic' symptoms in bipolar I, bipolar II and major depressive disorders. J. Affect Disord. 84, 159-166 (2005).

47. Fullerton, J. M. et al. Assessment of first and second degree relatives of individuals with bipolar disorder shows increased genetic risk scores in both affected relatives and young At-Risk Individuals. Am. J. Med. Genet. B: Neuropsychiatr. Genet. 168, 617-629 (2015).

48. Tondo, L., Lepri, B. \& Baldessarini, R. J. Suicidal risks among 2826 Sardinian major affective disorder patients. Acta Psychiatr. Scand. 116, 419-428 (2007).

49. Antypa, N., Antonioli, M. \& Serretti, A. Clinical, psychological and environmental predictors of prospective suicide events in patients with Bipolar Disorder. J. Psychiatr. Res. 47, 1800-1808 (2013).

50. Song, J. Y. et al. Assessment of risk factors related to suicide attempts in patients with bipolar disorder. J. Nerv. Ment. Dis. 200, 978-984 (2012).

51. Goffin, K. C. et al. Different characteristics associated with suicide attempts among bipolar I versus bipolar II disorder patients. J. Psychiatr. Res. 76, 94-100 (2016).

52. Tidemalm, D., Haglund, A., Karanti, A., Landén, M. \& Runeson, B. Attempted suicide in bipolar disorder: Risk factors in a cohort of 6086 patients. PLOS ONE 9, e94097 (2014).

53. Novick, D. M., Swartz, H. A. \& Frank, E. Suicide attempts in bipolar I and bipolar II disorder: a review and meta-analysis of the evidence. Bipolar Disord. 12, 1-9 (2010).

54. Forstner A. J. et al. Whole-exome sequencing of 81 individuals from 27 multiply affected bipolar disorder families. Transl. Psychiatry 10, 57 (2020).

55. Courtois, E. et al. Contribution of common and rare damaging variants in familial forms of bipolar disorder and phenotypic outcome. Transl. Psychiatry 10, 124 (2020). 